\title{
Educating Patients on Unnecessary Antibiotics: Personalizing Potential Harm Aids Patient Understanding
}

\author{
Benjamin J. Miller, BS, Kathryn A. Carson, ScM, and Sara Keller, MD, MPH, MSPH
}

Introduction: Antibiotic resistance is a public health emergency fueled by inappropriate antibiotic use. Public education campaigns often focus on global antibiotic resistance or societal harm of antibiotic misuse. There has been little research into what messages have the greatest impact on patient preferences for nonindicated antibiotics in ambulatory clinics.

Methods: We administered a survey at a primary care clinic in Baltimore, MD. A total of 250 participants rated 18 statements about potential harm from antibiotics on how each statement changed their likelihood to request antibiotics for an upper respiratory tract infection. Statements focused on potential harm either to the individual, to contacts of the individual, to society, and related or not to antibiotic resistance. Initial and final likelihood of requesting antibiotics was measured, and the impact of the statements in each category were compared using general linear models and Wilcoxon rank sum or Kruskal-Wallis tests.

Results: All statements decreased patient likelihood to request antibiotics. Statements about harm to the individual or contacts of the individual decreased participant likelihood to request antibiotics significantly more than statements about societal harm of antibiotic misuse. Statements not discussing antibiotic resistance decreased participant likelihood of requesting antibiotics significantly more than statements discussing antibiotic resistance. Overall likelihood to request antibiotics decreased after the survey by 2.2 points on an 11-point Likert scale $(P<.001)$.

Conclusion: When dissuading patients from requesting nonindicated antibiotics, providers and public health campaigns should focus on potential harm of nonindicated antibiotics to the individual rather than societal harm or antibiotic resistance. (J Am Board Fam Med 2020;33:969-977.)

Keywords: Ambulatory Care, Anti-Bacterial Agents, Anti-Bacterial Drug Resistance, Anti-Microbial Stewardship, Baltimore, Health Communication, Health Promotion, Linear Models, Patient Preference, Primary Health Care, Public Health, Surveys and Questionnaires

\section{Introduction}

While progress has been made in antibiotic stewardship, antibiotic resistance is increasing at an alarming rate. Antibiotic-resistant organisms cause more than 2.8 million infections and 35,000 deaths per year

This article was externally peer reviewed.

Submitted 11 May 2020; revised 10 July 2020; accepted 13 July 2020.

From the School of Medicine, Johns Hopkins, Baltimore, MD (BJM); Department of Epidemiology, Johns Hopkins Bloomberg School of Public Health, Baltimore, MD (KAC); Infectious Disease, Johns Hopkins Medicine, Baltimore, $\mathrm{MD}$ (SK).

Funding: None.

Conflict of interest: None.

Corresponding author: Sara Keller, MD, MPH, MSPH, Johns Hopkins University School of Medicine, $550 \mathrm{~N}$ Broadway St Suite 405, Baltimore MD 21287 (E-mail: skeller9@jhmi.edu). in the United States. ${ }^{1}$ In 2015, approximately 47 million antibiotic courses were prescribed for infections not needing antibiotics. ${ }^{1}$

Many interventions aimed at improving antibiotic use in ambulatory settings have focused on clinician behavior. However, clinicians cite perceived patient desire for antibiotics, impact of patient satisfaction scores, time demands, or lack of patient understanding about negative impacts of harmful antibiotic use as several reasons they may prescribe antibiotics for conditions where they are not indicated, such as for upper respiratory infections (URIs). ${ }^{2,3}$ Studies have shown that when physicians perceive that patients expect antibiotics, the physician is 10 times more likely to prescribe an antibiotic. ${ }^{2,4}$

To decrease unnecessary antibiotic prescriptions, clinicians may benefit from an improved understanding 
of how to communicate with patients about antibiotics. Patients may not appreciate the negative impact of taking nonindicated antibiotics, ${ }^{3}$ and it is unclear what information clinicians could provide to patients that has the greatest impact on patient desire for antibiotics. There is limited data on the effectiveness of specific language meant to dissuade patients' desire and requests for antibiotics for nonindicated conditions. While public health campaigns have often focused on global antibiotic resistance, little research has directly compared this approach with more personal messaging. ${ }^{5,6}$

The purpose of this study was to determine which statements most deter requests for antibiotics for nonindicated conditions by effectively communicating the risks associated with antibiotics. We hypothesized that statements about potential harm to the individual patient or contacts of the individual patient from unnecessary antibiotics would decrease participant desire for antibiotics more than statements about societal harm from nonindicated antibiotics.

\section{Methods}

\section{Survey Instrument}

We developed a survey with 18 statements in 4 categories. Participants rated each statement based on the impact on patient likelihood of requesting antibiotics for a URI. Each statement described a potential harm caused by antibiotics. Eight statements described potential harm to the individual taking the antibiotics, 4 statements described potential harm to people close to the individual taking the antibiotics, and 6 statements described potential harm to society. In addition, 8 of the statements discussed harm caused by antibiotic resistance while 10 of the statements did not discuss antibiotic resistance. To increase readability, statements were edited to use short sentences, words with few syllables, and clear sentence structure. The average Flesch-Kincaid readability score was grade 10.6. The survey was piloted with 10 adults: 5 female and 5 male, with ages ranging from 19 to 58 years and education level ranging from high school completion to advanced degree completion. Statements were refined for clarity and were narrowed from 24 to 18 in number based on pilot feedback, with the deletion of questions that were reported to be the most confusing or repetitive (Appendix).
Participation was requested by visual signage. Surveys were delivered in random question order either on article $(n=40)$ or an electronic tablet using the Qualtrics survey platform $(n=210)$, based on participant preference. Participants read each statement and then rated on a 11-point Likert scale how that statement changed their likelihood to request antibiotics for a URI. A "0" meant the statement made the patient much less likely to request antibiotics and " 10 " meant the statement made them much more likely to request antibiotics. We utilized an 11 point Likert scale to allow for a neutral choice (5), and since we anticipated most responses would fall on the "less likely" side of neutral, 5 options on either side of neutral provided more distinction between choices than 3 or 4 options. We wished to keep a 0 to 10 scale with neutral as 5 as this is the way people are used to seeing surveys. However to report the data we transposed the neutral " 5 " value to a $\delta$ Mean of " 0 ," the prior value of " 0 " to a $\delta$ mean of " -5 ," and the prior score of " 10 " to " 5 ." $\delta$ means more clearly display that the most effective statements had a large negative $\delta$ mean, while less effective statements had a less negative $\delta$ mean. Before and after reading the 18 statements, participants were asked about their overall likelihood of requesting antibiotics for a URI.

\section{Population}

Our survey was available to adults in the waiting room of an outpatient internal medicine and obstetrics and gynecology practice in Baltimore, $\mathrm{MD}$ on weekdays between July 20, 2018 and July 27, 2018. Participants were not required to be patients of the clinic (for example, they could have been a family member waiting with a clinic patient). Participants received a $\$ 10$ gift card on completion of the survey.

\section{Statistical Analysis}

Many of the survey response data were not normally distributed. Therefore, results were summarized using both nonparametric (medians, interquartile ranges) and parametric (means, standard deviations) statistics. Group comparisons were made using Wilcoxon rank sum or Kruskal-Wallis tests. General linear models regression was used to estimate the least squares means of the change in the likelihood of requesting antibiotics following the 18 statements. Statistical analysis was performed using SAS version 9.4 (SAS Institute, 
Inc., Cary, NC). All tests were 2-sided, and significance was set at $P<.05$.

\section{Results}

Of 1150 total adult patients in clinic and an unknown number of family members or others accompanying these patients during the 6-day course of the survey administration, 250 adults took the survey. Researchers were not allowed to approach any patients, and the 250 participants approached only after seeing signage. These 250 people were out of an unknown number of the 1150 patients in clinic who actually saw and read the signage. Most participants were women $(n=184$, $73.6 \%)$, most had completed at least some college or trade school $(\mathrm{n}=140 ; 56 \%)$, and most were African American ( $\mathrm{n}=152 ; 61 \%$; Table 1$)$.

The initial likelihood of requesting antibiotics for a URI like illness was a raw mean score of 5.3 (meaning slightly more likely to request antibiotics than not, as "5" meant "I may or may not request antibiotics"). After participants completed the survey, this likelihood decreased to a raw mean of 3.1 (3 meant "I probably will not request antibiotics," $P<.001)$ for a mean $\delta$ of -1.9 . All statements reduced participant likelihood of requesting antibiotics (Table 2). The most impactful statement was "Taking antibiotics can hurt your body's natural defenses. This makes it easier for you to get another infection" with a change from neutral (5) in mean likelihood of requesting antibiotics (mean $\delta$ ) of -2.56 while the least effective statement was "Antibiotic resistance costs the US between $\$ 20$ to 35 billion each year" with a $\delta$ mean of -1.35 .

Statements about both individual harm and harm to contacts of an individual had a significantly greater reduction in likelihood of requesting antibiotics ( $\delta$ mean, -2.30 and -2.18 , respectively) than statements about harm to society $(\delta$ mean, -1.80 ; $P<.001$ for both individual harm and harm to contacts of an individual compared with society; Table $3)$. Statements about individual harm ( $\delta$ mean, $-2.30)$ and harm to others close to an individual $(\delta$ mean, -2.18) did not significantly differ in their impact on likelihood of requesting antibiotics $(P=.11)$. Statements discussing antibiotic resistance $(\delta$ mean, $-1.91)$ led to a smaller reduction in likelihood to request antibiotics than statements not discussing antibiotic resistance $(\delta$ mean, $-2.26 ; P<.001)$. This difference was seen in each category of statement; that is, within the individual harm category, statements discussing antibiotic resistance were less effective than statements not discussing antibiotic resistance.

Each demographic group rated individual harm statements as most effective, followed by statements about harm to others and then harm to society (Table 3). Within each category, statements had slightly different rankings, such as women rating statements involving the harm of antibiotic misuse on a pregnant woman's child as more impactful than men did. For men, the most impactful statement was, "Taking antibiotics can hurt your body's natural defenses. This makes it easier for you to get another infection," ( $\delta$ mean, -2.30$)$ and the second most impactful was, "By changing your normal gut bacteria, antibiotics can cause allergies, asthma, and stomach problems" ( $\delta$ mean, -2.16$)$.

\section{Discussion}

The results suggest that providers should focus on individual harm or harm to others close to an individual and avoid discussing societal harm. Further, providers may wish to avoid focusing on antibiotic resistance as discussing antibiotic resistance did not decrease participant desire for antibiotics as much as other statements. Patients likely care the most about harm that they can both understand and apply to their own life.

All statements decreased participant likelihood of requesting antibiotics, and participants were less likely to request antibiotics after completing the questionnaire. Clinicians are more likely to prescribe a nonindicated antibiotic when they perceive that patients expect one., ${ }^{2,4}$ Clinician communication with patients about antibiotics can increase patient awareness of antibiotic resistance and understanding of appropriate indications for an antibiotic. ${ }^{20}$ Improving patient expectations around antibiotic use may decrease patient requests for antibiotics. Our study provides evidence that using specific statements may impact patient desire for antibiotics and provides guidance as to which of these statements are the most impactful.

Public education campaigns have frequently focused on the spread of antibiotic resistance. ${ }^{5,6}$ Our data suggest that this approach may not be the most effective messaging to convince an individual why they should not request nonindicated antibiotics. Antibiotic resistance is a complex topic that is poorly understood by the public. ${ }^{21}$ For example, even though $92 \%$ of the American public agrees that inappropriate antibiotic 
Table 1. Characteristics of 250 Adult Participants in a Survey about Harms of Antibiotic Use

\begin{tabular}{lc}
\hline Characteristic & $\begin{array}{c}\text { Count } \\
(\%)\end{array}$ \\
\hline Sex & \\
$\quad$ Male & $64(25.6)$ \\
Female & $184(73.6)$ \\
Other & $2(0.8)$ \\
Age, years & \\
18 to 24 & $18(7.2)$ \\
25 to 34 & $70(28.0)$ \\
35 to 44 & $43(17.2)$ \\
45 to 54 & $40(16.0)$ \\
55 to 64 & $40(16.0)$ \\
$65+$ & $39(15.6)$ \\
Education & \\
High school graduate or less & $54(21.6)$ \\
Any college or trade school up to bachelor's & $140(56.0)$ \\
degree & \\
Master's degree or higher & $56(22.4)$ \\
Race/ethnicity & \\
White (non-Hispanic/Latino) & $69(27.6)$ \\
Black or African American & $152(60.8)$ \\
All others & $29(11.6)$ \\
Living situation & \\
I live with family members or roommates & $190(76.0)$ \\
I live by myself & $60(24.0)$ \\
\hline
\end{tabular}

use contributes to antibiotic resistance, only $30 \%$ believes that antibiotic resistance is a problem. ${ }^{3}$ In administering our study, several study participants asked the study team member questions after the survey about whether antibiotic resistance was a positive or negative characteristic. Antibiotic resistance may be a complex topic to explain and to comprehend, so statements focusing on antibiotic resistance may not be as effective as statements focusing on other harms in talking to patients.

Statements focusing on harm to the individual had the greatest impact on patient likelihood to request antibiotics. It is possible that participants did not see how a global problem impacted them personally. While no research has directly measured patient consideration of individual harm from antibiotics in requesting antibiotics, recent work has shown that even when patients understand that antibiotics only treat bacteria and not viruses, they still believe that antibiotics are so low risk for causing side effects that they may believe that the antibiotics are more likely to help than hurt symptoms of a URI. ${ }^{21}$ In assessing evidence to this effect, recent public health campaigns have shifted toward personalizing the harm of antibiotic resistance such as by discussing possible harms such as Clostridioides difficile, allergic reactions, and antibiotic resistant infections. ${ }^{1}$ Our study suggests that this type of information is impactful when provided in a series of short statements. Making antibiotic misuse personal may be the most effective strategy in convincing patients why they should not request nonindicated antibiotics. ${ }^{21}$

In considering how best to dissuade a patient from nonindicated antibiotics, clinicians should keep in mind that different types of statements may have more of an impact on members of some demographic groups than others. For example, statements about the impact of pregnant women taking antibiotics on the development of babies had a greater impact on women than men. Level of educational attainment also impacted statement impact: those with a lower level of education were more likely to both initially desire antibiotics and be dissuaded by the statements than other groups. Providing education about potential harm may be most effective for those with lower levels of education.

Our quantitative examination into antibiotic resistance communication and motivations for patients to want antibiotics is novel. The relatively large study population included a high proportion of African American patients which matched the local community's demographics and increases study applicability to similar populations. A larger multi-site study would increase generalizability of results.

The study had several limitations. Generalizability is limited as it was conducted at a single site. In addition, as not every potential patient could be included in the study, the participants were not randomly selected, and participants may have been motivated to varying degrees by the $\$ 10$ gift card provided, there was the possibility of response bias. Researchers were not allowed to approach any potential participants about the study and had to wait for patients to approach after seeing signage. The inability to offer the survey to every patient in clinic, or to determine how many of the 1150 patients in clinic during survey administration saw and read the advertising signage excludes the denominator necessary to calculate a response rate. The survey's reading level was relatively high, driven by the words, "antibiotic" and "resistance." While this could have impacted participant understanding of the statements, distributing this survey without including the words, "antibiotic" 
Table 2. Change in Likelihood of Requesting Antibiotics for upper respiratory infections (URI)-like Symptoms after Reading Each Statement about Potential Harm of Nonindicated Antibiotics

\begin{tabular}{|c|c|c|c|c|}
\hline Statement & $\begin{array}{l}\Delta \text { Mean Likelihood } \\
\text { (Standard Deviation) }\end{array}$ & $\begin{array}{l}\text { Median } \\
\text { (Interquartile Range) }\end{array}$ & Statement Category & $\begin{array}{l}\text { Discusses Antibiotic } \\
\text { Resistance? }\end{array}$ \\
\hline $\begin{array}{l}\text { "Taking antibiotics can hurt } \\
\text { your body's natural defenses. } \\
\text { This makes it easier for you } \\
\text { to get another infection."1,7 }\end{array}$ & $-2.56(2.14)$ & $-3(-5$ to -1$)$ & Individual & No \\
\hline $\begin{array}{l}\text { "Antibiotics can change how } \\
\text { babies grow inside pregnant } \\
\text { women." } 8,9\end{array}$ & $-2.54(2.28)$ & $-3(-5$ to 0$)$ & Others close to you & No \\
\hline $\begin{array}{l}\text { "By changing your normal gut } \\
\text { bacteria, antibiotics can cause } \\
\text { allergies, asthma, and } \\
\text { stomach problems." } 8,10,11\end{array}$ & $-2.54(2.21)$ & $-3(-5$ to -1$)$ & Individual & No \\
\hline $\begin{array}{l}\text { "Antibiotics can make you } \\
\text { more likely to be obese } \\
\text { (fat)."12 }\end{array}$ & $-2.51(2.28)$ & $-3(-5$ to 0$)$ & Individual & No \\
\hline $\begin{array}{l}\text { "Pregnant women who take } \\
\text { antibiotics are more likely to } \\
\text { have babies with asthma."," }\end{array}$ & $-2.50(2.33)$ & $-3(-5$ to 0$)$ & Others close to you & No \\
\hline $\begin{array}{l}\text { "Antibiotics can cause bad } \\
\text { bacteria to overgrow in your } \\
\text { gut. This can cause diarrhea } \\
\text { and belly pain." }\end{array}$ & $-2.47(2.17)$ & $-3(-5$ to -1$)$ & Individual & No \\
\hline $\begin{array}{l}\text { "In the future, antibiotic } \\
\text { resistance will cause more } \\
\text { deaths than cancer and } \\
\text { diabetes combined." }\end{array}$ & $-2.42(2.23)$ & $-3(-5$ to 0$)$ & Society & Yes \\
\hline $\begin{array}{l}\text { "Resistant bacteria can still be } \\
\text { found in your gut } 4 \text { years } \\
\text { after taking antibiotics." }\end{array}$ & $-2.30(2.25)$ & $-3(-4$ to 0$)$ & Individual & Yes \\
\hline $\begin{array}{l}\text { "A single dose of antibiotics } \\
\text { makes the bacteria in your } \\
\text { body more resistant to } \\
\text { treatment." }\end{array}$ & $-2.12(2.26)$ & $-2(-4$ to 0$)$ & Individual & Yes \\
\hline $\begin{array}{l}\text { "Antibiotics kill your normal } \\
\text { gut bacteria. This can cause } \\
\text { bad bacteria to overgrow."12 }\end{array}$ & $-1.99(2.35)$ & $-2(-4$ to 0$)$ & Individual & No \\
\hline $\begin{array}{l}\text { "Antibiotic resistant bacteria } \\
\text { cause over } 2 \text { million illnesses } \\
\text { and } 23,000 \text { deaths in the US } \\
\text { each year.", }\end{array}$ & $-1.96(2.45)$ & $-2(-4$ to 0$)$ & Society & Yes \\
\hline $\begin{array}{l}\text { "Each antibiotic-resistant } \\
\text { infection costs up to } \$ 30,000 \\
\text { more to treat than other } \\
\text { infections." }\end{array}$ & $-1.93(2.30)$ & $-2(-4$ to 0$)$ & Society & Yes \\
\hline $\begin{array}{l}\text { "Taking antibiotics can cause } \\
\text { you to get a yeast } \\
\text { infection." } 18\end{array}$ & $-1.90(2.46)$ & $-2(-4$ to 0$)$ & Individual & No \\
\hline $\begin{array}{l}\text { "Resistant bacteria can spread } \\
\text { between people." }\end{array}$ & $-1.85(2.66)$ & $-2(-4$ to 0$)$ & Others close to you & Yes \\
\hline $\begin{array}{l}\text { "Antibiotics are the most } \\
\text { common cause of Emergency } \\
\text { Room visits for drug } \\
\text { reactions in children."7 }\end{array}$ & $-1.83(2.33)$ & $-2(-4$ to 0$)$ & Others close to you & No \\
\hline $\begin{array}{l}\text { "One in } 5 \text { people who take an } \\
\text { antibiotic in a hospital will } \\
\text { have a side effect." }\end{array}$ & $-1.72(2.25)$ & $-2(-4$ to 0$)$ & Society & No \\
\hline
\end{tabular}

Continued 
Table 2. Continued

\begin{tabular}{lcccc}
\hline Statement & $\begin{array}{c}\Delta \text { Mean Likelihood } \\
\text { (Standard Deviation) }\end{array}$ & $\begin{array}{c}\text { Median } \\
\text { (Interquartile Range) }\end{array}$ & Statement Category & $\begin{array}{c}\text { Discusses Antibiotic } \\
\text { Resistance? }\end{array}$ \\
\hline $\begin{array}{l}\text { "In some countries like India, } \\
\text { people in hospitals have } \\
\text { infections so resistant that } \\
\begin{array}{l}\text { antibiotics can't treat } \\
\text { them."19 }\end{array}\end{array}$ & $-1.40(2.60)$ & $-1(-4$ to 0$)$ & Society & Yes \\
$\begin{array}{l}\text { "Antibiotic resistance costs the } \\
\begin{array}{l}\text { US between } \$ 20 \text { and } \$ 35 \\
\text { billion each year." }\end{array}\end{array}$ & $-1.35(2.28)$ & $-1(-3$ to 0$)$ & Society & Yes \\
\hline
\end{tabular}

For the mean $\delta$ displayed, a " -5 " meant the statement made the patient much less likely to request antibiotics, "0" meant the statement did not change their likelihood, and " 5 " would mean the statement made them much more likely to request antibiotics.

and "resistance," would have not been reflective of public health educational campaigns to decrease antibiotic resistance. ${ }^{1,7}$ This study should be validated by administration of a similar survey at multiple sites, and could be offered or administered to every patient at participating sites to improve study validity.

\section{Conclusions}

Patient expectation of an antibiotic increases physician prescription by as much as 10 times, ${ }^{4}$ and while public education campaigns have demonstrated an ability to increase patient knowledge around appropriate antibiotic usage, ${ }^{5,20}$ little research exists addressing how best to directly dissuade a patient who is requesting nonindicated antibiotics from their request. Prior public health campaigns addressing patients have focused on antibiotic resistance and large-scale societal harm. Our research supports the approach suggested by the Centers for Disease Control and Prevention and other stakeholder organizations to shift outpatient clinic and overall discussion toward a more individual-oriented conversation focused on harm to the individual. ${ }^{1}$ Our research provides a list

Table 3. Mean $\Delta$ of Likelihood to Request Antibiotics Overall and by Category and Participant Characteristics

\begin{tabular}{|c|c|c|c|c|c|c|}
\hline $\begin{array}{l}\text { Statement } \\
\text { Category }\end{array}$ & Individual & Close Contact & About Society & About Resistance & Not about Resistance & All Statements \\
\hline $\begin{array}{l}\text { Total Sample } \\
\quad(\mathrm{n}=250)\end{array}$ & -2.30 & -2.18 & -1.80 & -1.91 & -2.26 & -2.10 \\
\hline \multicolumn{7}{|l|}{ Sex } \\
\hline $\operatorname{Men}(n=64)$ & -1.75 & -1.49 & -1.35 & -1.43 & -1.77 & -1.62 \\
\hline $\begin{array}{l}\text { Women } \\
\quad(\mathrm{n}=184)\end{array}$ & -2.81 & -2.42 & -2.95 & -2.08 & -2.43 & -2.28 \\
\hline \multicolumn{7}{|l|}{ Education } \\
\hline $\begin{array}{l}\text { HS graduate or } \\
\text { less }(n=54)\end{array}$ & -1.90 & -1.63 & -1.15 & -1.33 & -1.72 & -1.60 \\
\hline $\begin{array}{l}\text { Any college or } \\
\text { trade school up } \\
\text { to bachelor's } \\
\text { degree }(n=140)\end{array}$ & -2.30 & -2.33 & -1.86 & -1.95 & -2.33 & -2.16 \\
\hline $\begin{array}{l}\text { Master's degree } \\
\text { or higher } \\
(\mathrm{n}=56)\end{array}$ & -2.65 & -2.34 & -2.25 & -2.38 & -2.50 & -2.45 \\
\hline \multicolumn{7}{|l|}{ Race } \\
\hline Black $(\mathrm{n}=152)$ & -1.97 & -1.94 & -1.51 & -1.56 & -2.01 & -1.81 \\
\hline White (n = 69) & -2.69 & -2.41 & -2.13 & -2.32 & -2.53 & -2.44 \\
\hline Other $(\mathrm{n}=29)$ & -3.09 & -2.89 & -2.49 & -2.81 & -2.88 & -2.84 \\
\hline
\end{tabular}

HS, high school.

A mean $\delta$ " -5 " meant the statement made the patient much less likely to request antibiotics, " 0 " meant the statement did not change their likelihood, and "5" meant the statement made them much more likely to request antibiotics. 
of clear statements that clinicians can use in talking with patients about antibiotics. Clinicians and public health campaigns should focus on individual side effects or harm to others close to the patient when dissuading patients from nonindicated antibiotics and should not emphasize societal impacts or antibiotic resistance. Whenever possible, clinicians should use statements that are simple, understandable, and directly related to the patient.

To see this article online, please go to: bttp://jabfm.org/content/ 33/6/969.full.

\section{References}

1. Center for Disease Control and Prevention. Antibiotic resistance threats in the United States 2019 Atlanta, Georgia. Available from: https://www.cdc.gov/ drugresistance/pdf/threats-report/2019-ar-threatsreport-508.pdf. Accessed May 10, 2020.

2. Butler C, Rollnick S, Pill R, Maggs-Rapport F, Stott N. Understanding the culture of prescribing: qualitative study of general practitioners' and patients' perceptions of antibiotics for sore throats. BMJ 1998;317:637-42.

3. Carter RR, Sun J, Jump RL. A survey and analysis of the American public's perceptions and knowledge about antibiotic resistance. Open Forum Infect Dis 2016;3:ofw112.

4. Sirota M, Round T, Samaranayaka S, Kostopoulou O. Expectations for antibiotics increase their prescribing: causal evidence about localized impact. Health Psychol 2017;36:402-9.

5. Hwang TJ, Gibbs KA, Podolsky SH, Linder JA. Antimicrobial stewardship and public knowledge of antibiotics. Lancet Infect Dis 2015;15:1000-1.

6. O'Neill J. Antimicrobial resistance: tackling a crisis for the health and wealth of nations. review on antimicrobial resistance. 2014. Available from: https:// amr-review.org/sites/default/files/AMR\%20Review \%20Paper\%20-\%20Tackling\%20a\%20crisis\% 20for\%20the\%20health\%20and\%20wealth\%20of \%20nations_1.pdf Accessed December 8, 2019.

7. Center for Disease Control and Prevention. Antibiotic resistance threats in the United States. Atlanta: Report. 2013. Available from: https://www. cdc.gov/drugresistance/pdf/ar-threats-2013-508. pdf. Accessed December 8, 2019.

8. Russell SL, Gold MJ, Hartmann M, et al. Early life antibiotic-driven changes in microbiota enhance susceptibility to allergic asthma. EMBO Rep 2012;13:440-7.

9. Kashanian M, Mohtashami SS, Bemanian MH, Moosavi SAJ, Lakeh M. Evaluation of the associations between childhood asthma and prenatal and perinatal factors. Int J Gynecol Obstet 2017;137:290-4.
10. Lewis JD, Chen EZ, Baldassano RN, et al. Inflammation, antibiotics, and diet as environmental stressors of the gut microbiome in pediatric Crohn's disease. Cell Host Microbe 2015;18:489-500.

11. Nash M, Frank D, Friedman J. Early microbes modify immune system development and metabolic homeostasis-The "restaurant" hypothesis revisited. Front Endocrinol (Lausanne) 2017;8:349.

12. Mikkelsen KH, Allin KH, Knop FK. Effect of antibiotics on gut microbiota, glucose metabolism and body weight regulation: a review of the literature. Diabetes Obes Metab 2016;18:444-53.

13. Cheng G, Li Z, Dai X. Analysis of clostridium difficile associated diarrhea in pediatric patients with antibiotic-associated diarrhea. Zhonghua Er Ke Za Zhi 2015;53:220-4.

14. Jakobsson HE, Jernberg C, Andersson AF, SjölundKarlsson M, Jansson JK, Engstrand L. Short-term antibiotic treatment has differing long-term impacts on the human throat and gut microbiome. PLoS ONE 2010;5:e9836.

15. Khalil D, Hultin M, Rashid MU, Lund B. Oral microflora and selection of resistance after a single dose of amoxicillin. Clin Microbiol Infect 2016;22:949.e1-949.e4.

16. Filice GA, Nyman JA, Lexau C, et al. Excess costs and utilization associated with methicillin resistance for patients with Staphylococcus aureus infection. Infect Control Hosp Epidemiol 2010;31:365-73.

17. Mauldin PD, Salgado CD, Hansen IS, Durup DT, Bosso JA. Attributable hospital cost and length of stay associated with health care-associated infections caused by antibiotic-resistant gram-negative bacteria. AAC 2010;54:109-15.

18. Goncalves B, Ferreira C, Alves C, Henriques M, Azeredo J, Silva S. Vulvovaginal candidiasis: epidemiology, microbiology and risk factors. Crit Rev Microbiol 2016;42:905-27.

19. O'Neill J. Tackling drug-resistant infections globally: final report and recommendations. The review on antimicrobial resistance. 2016. Available from: https://amr-review.org/sites/default/files/160525_ Final\%20paper_with\%20cover.pdf. Accessed December 8, 2019.

20. Trepka MJ, Belongia EA, Chyou PH, Davis JP, Schwartz B. The effect of a community intervention trial on parental knowledge and awareness of antibiotic resistance and appropriate antibiotic use in children. Pediatrics 2001;107:E6-e6.

21. Broniatowski D, Klein E, Reyna V. Germs are germs, and why not take a risk? Patients' expectations for prescribing antibiotics in an inner-city emergency department. Med Decis Making 2015;35: $60-7$. 


\section{Appendix: Copy of Survey Administered}

"Thank you for taking this survey! You will be presented with a series of statements. All the information presented to you is factually true. Your completion of the survey will serve as your consent to be in this research study.

1. Please answer this question based on what you know about antibiotics.

If you feel sick, how likely are you to ask a doctor for antibiotics to help you feel better?

I definitely will not I probably will not I may or may not I probably will I definitely will ask for antibiotics ask for antibiotics ask for antibiotics ask for antibiotics ask for antibiotics

012345 (neutral) 678910

2. Antibiotics only fight infections caused by bacteria. They are very good at fighting bacterial infections. Like all drugs, they can be harmful and should only be used when necessary. Taking antibiotics when you have a virus will not help and could even hurt you. Antibiotics also make bacteria more resistant to them. This can make future infections harder to treat. This means that antibiotics might not work when you really need them. Because of this, it is important that you only take an antibiotic when it is necessary to treat your illness.

This survey discusses some of the problems antibiotics can cause if they are used when they are not needed. The following statements are all true.

On a scale of 0 to 10 , please rate whether reading this statement makes you more or less likely to ask for antibiotics. 0 means reading this statement makes you much less likely to ask for antibiotics. 5 means your likelihood of taking antibiotics has not changed from this statement. 10 means you are much more likely to ask for antibiotics after reading this statement. You can pick any number on the scale.

1. On a scale of 0 to 10 , please rate whether reading this statement makes you more or less likely to ask for antibiotics.

"Antibiotics kill your normal gut bacteria. This can cause bad bacteria to overgrow."

2. On a scale of 0 to 10 , please rate whether reading this statement makes you more or less likely to ask for antibiotics.

"Taking antibiotics can cause you to get a yeast infection."

3. On a scale of 0 to 10 , please rate whether reading this statement makes you more or less likely to ask for antibiotics.
"Antibiotics can make you more likely to be obese (fat)."

4. On a scale of 0 to 10 , please rate whether reading this statement makes you more or less likely to ask for antibiotics.

"Pregnant women who take antibiotics are more likely to have babies with asthma"

5. On a scale of 0 to 10 , please rate whether reading this statement makes you more or less likely to ask for antibiotics.

"In the future, antibiotic resistance will cause more deaths than cancer and diabetes combined."

6. On a scale of 0 to 10 , please rate whether reading this statement makes you more or less likely to ask for antibiotics.

"A single dose of antibiotics makes the bacteria in your body more resistant to treatment"

7. On a scale of 0 to 10 , please rate whether reading this statement makes you more or less likely to ask for antibiotics.

"Resistant bacteria can still be found in your gut 4 years after taking antibiotics."

8. On a scale of 0 to 10 , please rate whether reading this statement makes you more or less likely to ask for antibiotics.

"By changing your normal gut bacteria, antibiotics can cause allergies, asthma, and stomach problems."

Much less likely Unchanged Much more likely

012345678910

9. On a scale of 0 to 10 , please rate whether reading this statement makes you more or less likely to ask for antibiotics.

"Each antibiotic resistant infection costs up to $\$ 30,000$ more to treat than other infections."

10. On a scale of 0 to 10 , please rate whether reading this statement makes you more or less likely to ask for antibiotics.

"Antibiotic resistant bacteria cause over 2 million illnesses and twenty-three thousand deaths in the US each year."

11. On a scale of 0 to 10 , please rate whether reading this statement makes you more or less likely to ask for antibiotics.

"Resistant bacteria can spread between people."

12. On a scale of 0 to 10 , please rate whether reading this statement makes you more or less likely to ask for antibiotics.

"Taking antibiotics can hurt your body's natural defenses. This makes it easier for you to get another infection." 
13. On a scale of 0 to 10 , please rate whether reading this statement makes you more or less likely to ask for antibiotics.

"Antibiotic resistance costs the US between \$20 to 35 billion each year."

14. On a scale of 0 to 10 , please rate whether reading this statement makes you more or less likely to ask for antibiotics.

"Antibiotics can cause bad bacteria to overgrow in your gut. This can cause diarrhea and belly pain."

15. On a scale of 0 to 10 , please rate whether reading this statement makes you more or less likely to ask for antibiotics.

" 1 in 5 people who take an antibiotic in a hospital will have a side effect."

16. On a scale of 0 to 10 , please rate whether reading this statement makes you more or less likely to ask for antibiotics.

"In some countries like India, people in hospitals have infections so resistant that antibiotics cannot treat them"

17. On a scale of 0 to 10 , please rate whether reading this statement makes you more or less likely to ask for antibiotics.

"Antibiotics are the most common cause of Emergency Room visits for drug reactions in children."

18. On a scale of 0 to 10 , please rate whether reading this statement makes you more or less likely to ask for antibiotics.

"Antibiotics can change how babies grow inside pregnant women"

19. Thank you for completing the above questions!

After reading the information in this survey, please answer this question a second time.

"If you feel sick, how likely are you to ask a doctor for antibiotics to help you feel better?"

I definitely will not I probably will not I may or may not I probably will I definitely will ask for antibiotics ask for antibiotics ask for antibiotics ask for antibiotics ask for antibiotics
012345 (neutral) 678910

Finally, please complete these final anonymous demographic questions.

1. Please select your sex.

Male

Female

Other (Please specify)

2. Ethnicity origin (or Race): Please specify your ethnicity.

Asian/Pacific Islander

Black or African American

Hispanic or Latino

Native American or American Indian

White

Other (please specify)

3 . What is your age?

18 to 24 years old

25 to 34 years old

35 to 44 years old

45 to 54 years old

55 to 64 years old

65 to 74 years old

75 years or older

4. Education: What is the highest degree or level of school you have completed? If currently enrolled, highest degree received.

No schooling completed.

Nursery school to eighth grade.

Some high school, no diploma.

High school graduate, diploma or the equivalent (for example: GED).

Some college credit, no degree.

Trade/technical/vocational training.

Associate degree.

Bachelor's degree.

Master's degree.

Professional degree.

Doctorate degree.

5. Living situation:

I live with family members or roommates.

I live by myself. 